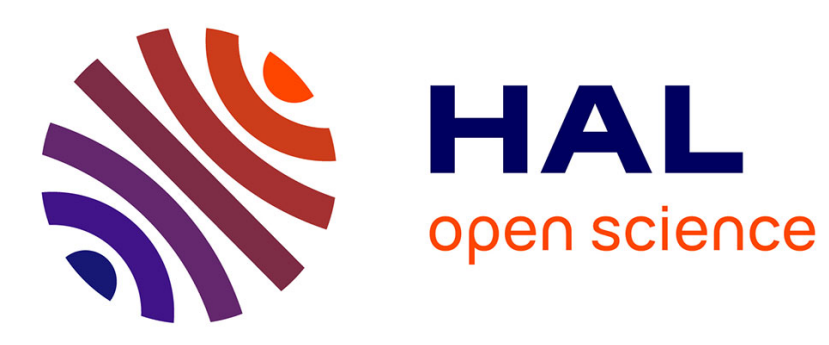

\title{
An appearance fuzzy sensor integrating a knowledge model
}

Emmanuel Schmitt, Vincent Bombardier, Patrick Charpentier, Raphaël Vogrig

\section{To cite this version:}

Emmanuel Schmitt, Vincent Bombardier, Patrick Charpentier, Raphaël Vogrig. An appearance fuzzy sensor integrating a knowledge model. 12th IFAC Symposium on Information Control Problems in Manufacturing, INCOM'2006, May 2006, Saint-Etienne, France. pp.111-116. hal-00087472

\section{HAL Id: hal-00087472 \\ https://hal.science/hal-00087472}

Submitted on 29 Sep 2006

HAL is a multi-disciplinary open access archive for the deposit and dissemination of scientific research documents, whether they are published or not. The documents may come from teaching and research institutions in France or abroad, or from public or private research centers.
L'archive ouverte pluridisciplinaire HAL, est destinée au dépôt et à la diffusion de documents scientifiques de niveau recherche, publiés ou non, émanant des établissements d'enseignement et de recherche français ou étrangers, des laboratoires publics ou privés. 


\title{
E. SCHMITT ${ }^{1,2}$, V. BOMBARDIER ${ }^{1}$, P. CHARPENTIER $^{1}$, R. VOGRIG $^{2}$
}

\author{
${ }^{1}$ Centre de Recherche en Automatique de Nancy (CRAN), CNRS UMR 7039 \\ Faculté des Sciences, Bd des Aiguillettes, BP 239, 54506 Vandoeuvre lès Nancy Cedex \\ ${ }^{2}$ LuxScan Technologies \\ ZARE Ouest, L-4384 Ehlerange, Luxembourg
}

\begin{abstract}
This article exposes a modeling process of expert's knowledge to improve a system of wooden board appearance classification. The aesthetic criteria for the classification are the color and the texture. The extraction of knowledge concerning these two notions is realized with the Natural language Information Analysis Method (NIAM). Then, to improve the current industrial system, we suggest to use this symbolic knowledge model to generate a numeric model. This numeric part is built thanks to a Fuzzy Rules based Inference System (SIF). Fuzzy Sets Theory is here well adapted in order to obtain not-disjointed result classes and to manipulate subjective or symbolic information. Finally, we propose to create our appearance sensor under the form of a “fuzzy sensor”. Copyright (C) INCOM2006.
\end{abstract}

Keywords: Knowledge modelling, Fuzzy sensor, Fuzzy rules, Color, Texture.

\section{INTRODUCTION}

Nowadays, the improvement of the production for the wood product industries (sawmill, furniture manufacturers ...) is a consequent stake. One task that wood product suppliers are currently faced with is the matching boards according to their appearance. In fact, the face of higher quality wooden objects (furniture, parquet floor ...) should have a uniform and continuous aspect according to an aesthetic criterion. Moreover, this kind of criterion is a very subjective one.

In order to ensure a control of performances and product quality, some sensors are developed. At present, the existing tools are not adapted to the complexity of current systems. For the mentioned problem: the wooden board classification according to the "aspect", we need to use sensors more and more sophisticated. These sensors have to sort wooden board with the same characteristics defined by the customer. That's means that all the board belonging to the same class must have similar "color" and "grain" in wooden terms. Thus, the "aspect" notion evoked by the customer is linked to a color aspect and texture aspect in the field of image processing.

In general, the color and the texture grading procedure if highly subjective and requires human intervention. The humans who validate the classification must understand information delivered by the system. That's why we introduce a novel approach integrating a knowledge model on the wooden boards appearance associated to the notion of fuzzy sensors. In fact, our study reinforces the notion of symbolic fuzzy sensors allowing to process more intelligently the data provided by the system. Through these terms, we considered three sections in this article. Firstly, we define a reliable and reproducible vision system. In a second time, we expose the knowledge models used in parallel with our classifier. Then, we explain our methodology of classification based on fuzzy inferences system for the color point of view. Finally, we expose some results showing the efficiency of our approach.

\section{INDUSTRIAL FRAMEWORK}

The work described here has been motivated by a collaborative effort between academia and industry. The Automatic Research Centre of Nancy (CRAN) is the academic partner and Luxscan Technologies, a start-up company based in the Luxembourg is the industrial partner. This study concerns the development of a sorting system for wooden boards according to the color and the texture. The recognition of the board appearance is realized in real time on production line. These lines may reach speeds of 180 meters of board length per minute.

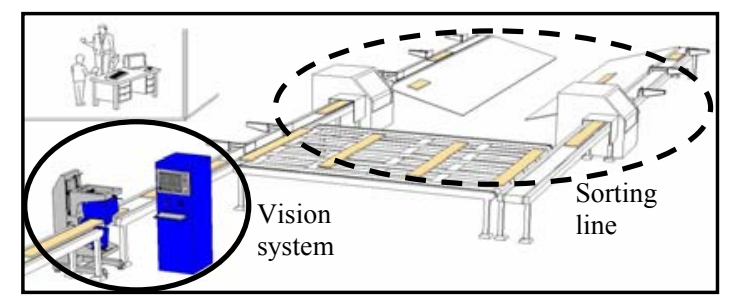

Fig. 1. Example of production line 
After the identification of the color and texture, the wood board is sent to a sorting line (Fig. 1). We use also the term of optimisation of the quality and the products quantity according to the customer needs.

The used vision system is made up essentially of one type of linear sensors: color cameras. This kind of sensor returns red, green and blue components of the signal. The signals are sampled on 256 levels. Then, they are processed to deliver useful characteristics for the board aspect determination. This attributes will be exposed in the following section.

The first question, which is appeared, was: "How could we define and characterize the appearance of a wood board?" To answer at this question, we decide to generate a knowledge model concerning the field of the wood industry.

\section{KNOWLEDGE MODELLING}

To characterize the aspect of a wood board, we decide to create a global model, which contains the notions of color and texture. This symbolic model concerns the formalization of the expert knowledge in the wood field and the vision field. To elaborate this model, we use the Natural Information Analysis Method (NIAM). To explain the results of the model, we use a derived formalism of NIAM: Object Role Modelling (ORM) (Halpin, 1998).

\subsection{Principles of NIAM/ORM method}

With the NIAM method, we distinguish two kinds of systems: the object system, the abstraction system. To represent these two systems, we use:

- The Lexical Object Type, also name LOT, for the object system. It corresponds to a part of the observable reality for which we want to collect information.

- The NO Lexical Object Type, also name NOLOT, for the abstraction system. It corresponds to the symbolic model of the object system. It is made up of objects and rules classes.

This method allows to express an expression of the knowledge in natural language. To complete the generated model, we can ask precise questions to the expert in relation with this method. By example: does a color have one or several hues? In that case, we introduce constraints of uniqueness and totality. The figure Fig. 2 explains this formalism.

\subsection{Wood expert knowledge modelling}

The model for the aspect is created thanks to a definition made by the wood expert in a natural language. The description of the appearance is the following:

"An aspect Dark Red is a combination of a red color and a very contrasted texture".
It exists the same kind of sentence for the texture and the color, but we expose here the example of the aspect.
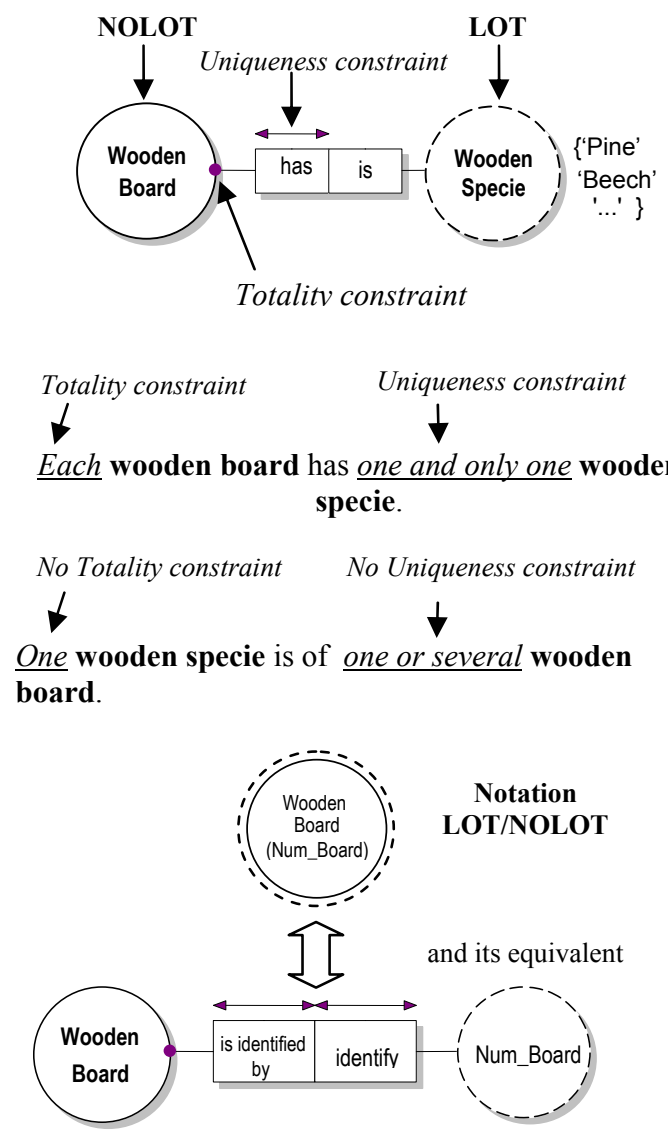

Fig. 2. Formalism of NIAM/ORM method

From the NIAM method, we can split this complex sentence in elementary sentences. The different parts follow the structure " $<$ subject $>, \quad<$ verb $>$, $<$ complement $>$ ". For our example, we obtain the following results:

- "An aspect represented by the type $\{$ Dark Red $\}$ has a color represented by a color value $\{$ Red $\}$. “

- "An aspect represented by the type $\{$ Dark Red $\}$ has a texture represented by an texture value \{Very Contrasted $\}$."

The figure Fig. 3 illustrates the formalization of the previous elementary sentences.

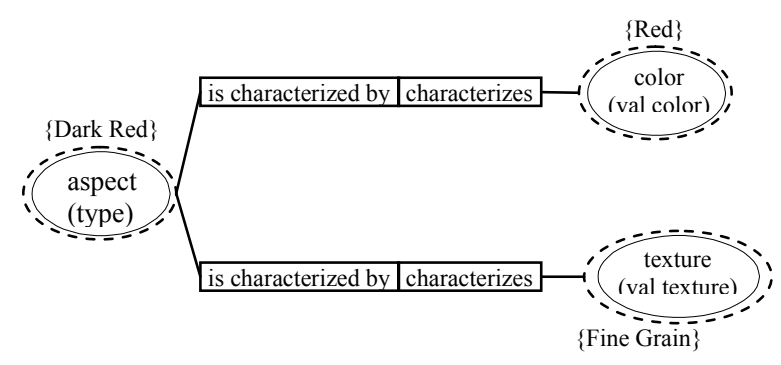

Fig. 3. Partial NIAM formalization of the aspect Dark Red

It is possible to decompose the color item and the texture item in the same way. Thanks to these decompositions, we can expose the next level of our 
knowledge model in a generic way. In the case of the color, we divided this notion in luminance and chrominance. By including the uniqueness and totality constraints, we obtain a generic model to represent the color (Fig. 4). In the same way, we can create a generic model to characterize the texture of a wood board (Fig. 5).

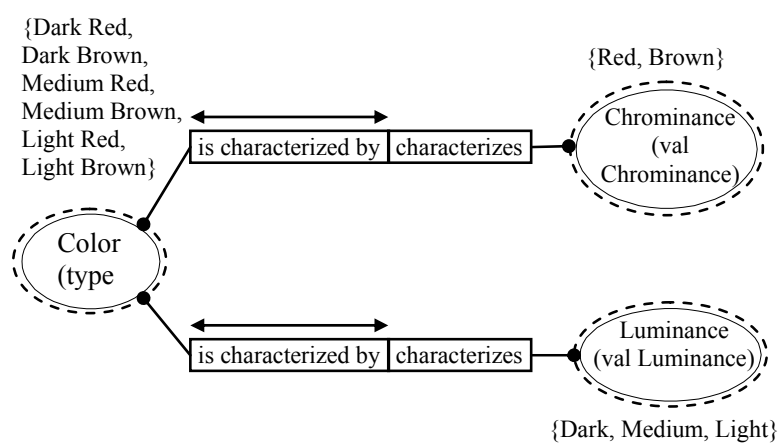

Fig. 4. Generic NIAM model of the color

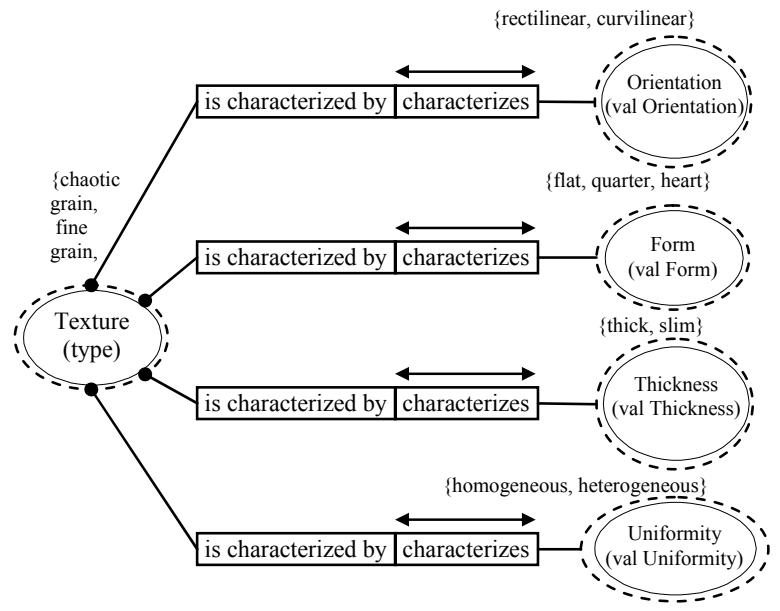

Fig. 5. Generic NIAM model of the texture

\subsection{Vision expert knowledge modelling}

In this part, we only present the model concerning the color. This work on the texture notion is in progress.

The knowledge provided by the vision expert concerns essentially the color identification system. In fact, we want to precise the useful attributes to quantify the characteristics of the color. The principle is the same than the one exposed in section 3.2. But, in this context, we represent the color trough vision parameters. Like this, a color is defined as following:

"A Luminance of a wood board is represented by the attribute giving the intensity level in the picture. More the intensity is dark more the attribute value is small."

Then, the elementary sentences are:

- "A Luminance characterized by an intensity level $\{$ dark $\}$ is represented with the intensity level value \{small\}."

- "A Luminance level value \{small\} represents an intensity characterized by an intensity level $\{$ dark $\}$."
We realized the same work with the Chrominance notion, but we cannot expose the NIAM model for this attribute for confidential reasons.

\section{FUZZY SENSOR FOR COLOR CLASSIFICATION}

The colors, which are classified, are very closed. We use numeric data, which are sent by the recognition module. These data are given in the form of a membership coefficient of one color class. They allow to interpret the results and to keep the fuzzy value provided by the recognition system. In fact, we would like to attach a numeric data to symbolic terms for all the production line. This kind of language could improve the human comprehension of the system. For a manufacturer, it is easier to work with word and not with incoherent numbers.

That's why, we decide to develop our system under the form of an appearance fuzzy sensor (Fig. 6) (Benoit, and Foulloy, 2000).

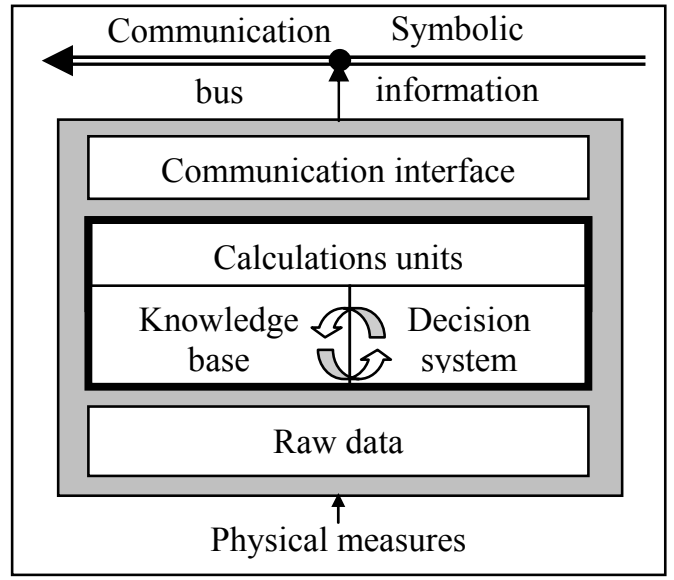

Fig. 6. Structure of the developed fuzzy sensor

The primary interest to use the concept of fuzzy sensor is to keep a symbolic result in output of the classification system (Fig. 7).

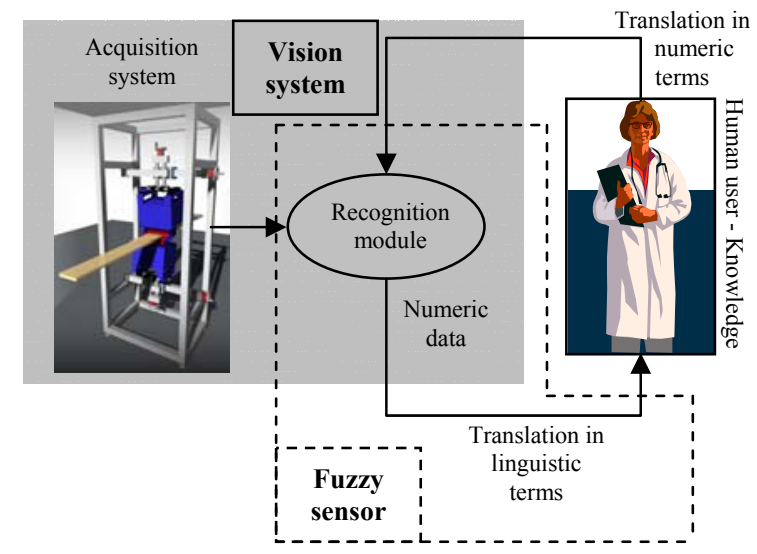

Fig. 7. Fuzzy sensor implementation in vision system

Then, we will obtain a better interpretability of results. By taking as example the color, a noninitiated person will better understand words to 
define a color rather than a numeric value. For the adjustment of the vision system, it is easier for a noninitiated person to use linguistic terms. This human is surely incapable to convert his needs in numeric data.

Moreover, the color perception is gradual and subjective. In fact, our system has to classify notdisjointed classes. It exist confusion zones between two or more colors (region between dark red and medium red by example).

To create the fuzzy sensor, we need to connect the symbolic field and the numeric field. This link is realized thanks to a method based on fuzzy subsets theory (Zimmermann, 1983). This process allows to compare the symbolic knowledge model of experts with the results of the classification system. Galichet and Foulloy (2003) propose several structures to generate collaboration between the numeric and symbolic processing implemented with fuzzy linguistic systems.

\subsection{Fuzzy Inferences System}

Two reasoning types using fuzzy rules exist:

- the abductive reasoning, where we search to obtain information on the input $\mathrm{X}$ (pixel values) from the output variable Y (the color classes);

- the deductive reasoning, where want to deduce Y from the values of $\mathrm{X}$.

For our problem, we consider the second case corresponding to the Modus Ponens. Zadeh (1973) has generalized this process with the combinationprojection principle of two fuzzy rules representing the rule and the input (Klir, and Yuan, 1995). Two cases are available to classify the generated rules: conjunctives rules and implicative rules (Dubois, and Prade, 1992, 1996). This dichotomy is explained also in a "cultural" point of view. For the implicative rules, the reasoning is governed by the knowledge. The more information we will have on the product, the more the results will be precise. In our case, we are located in the low level part of the image processing, so we don't have enough rich information to use this kind of rules. In fact, the conjunctive rules result from the data analysis field. That's why we have chosen a conjunctive parallel rules mechanism.

Two main models can be used to build such parallel mechanism: Larsen model and Mamdani model. The difference between them is made according to the TNorm choice for implicative operator. Finally, we have chosen an inference mechanism based on Larsen model, because the Product is more adapted than the Minimum T-Norm in our case. In fact, by using the Product, we allow a non-linear splitting of input variables spaces. After this premises processing, we combine the different partial results with a disjunction operator (T-Conorm): the Maximum. In our case, we don't want to generate a composition law but use a pseudo-implication of
Larsen. The color classification method must allow to use the numeric data distributed by our sensors. It exist lots of methods providing automatically fuzzy rules from a data set (Cordon, et al., 1999; Berthold, 2003). We have decided to use the algorithm developed by Ishibuchi, et al. (1992), because it replies to our needs previously evoked. It uses an iterative inference mechanism based on Larsen model. This algorithm decomposes of three parts: the input fuzzification, the rules generation and the numeric model adjustment. Then, the obtained rule set is used to process the recognition.

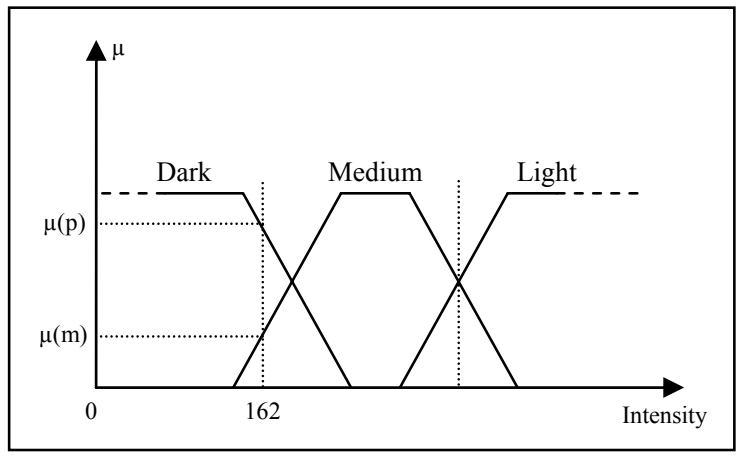

Fig. 8. Intensity level fuzzification in 3 terms

Attributes fuzzification. The fuzzification consists on a decomposition of the parameters on several terms. Each part of this decomposition is expressed by a word of the natural language. By example, the intensity level can be "light", "medium" or "dark". From this decomposition, membership functions are described thanks to the analysis of samples set used for the numeric model generation. An example of decomposition is given by the figure Fig. 8 .

Linguistic rules generation. After this first step, we create the numeric model. The used method is based on a supervised training system. It allows to generate fuzzy rules "IF ... THEN ...". By example, "IF hue (big) AND IF intensity (big) THEN color (white)". We use a training samples set to create the model. In our case, the samples used for the supervised training are selected in harmony with the customers. In fact, this algorithm describes the color perception of the system. Generally speaking, if we consider two parameters in input and one color in output, the fuzzy rule follows the form:

"IF $x_{1}$ is $A_{i}$ AND IF $x_{2}$ is $A_{k}$ THEN $y_{1}$ is in the color class $\mathrm{C}_{\mathrm{n}}$ "

where $\mathrm{x}_{1}$ and $\mathrm{x}_{2}$ are the input attributes

$\mathrm{y}_{1}$ is the output data

$A_{i}$ and $A_{k}$ are the fuzzy subsets

$\mathrm{C}_{\mathrm{n}}$ is the class of the $\mathrm{n}^{\text {th }}$ color

From that, the numeric results provided by the classifier module will be represented and interpreted as symbolic results.

Model adjustment. We used the iterative part of this algorithm for the model adjustment step (Ishibuchi, et al., 1997). In fact, we adjust the splitting of the 
representation space of the input attributes according to the results given by the training samples set.

The supervised training step must be realized with lots of attentions by selecting representative samples of each color classes. The general principle of the recognition module is shown Fig. 9.

Other methods have been used to classify wooden board according to the color (Kline, et al., 1997). In the next sections, we compared our methodology versus these classical methods, which don't take into account the gradual aspect of our color classification problem.

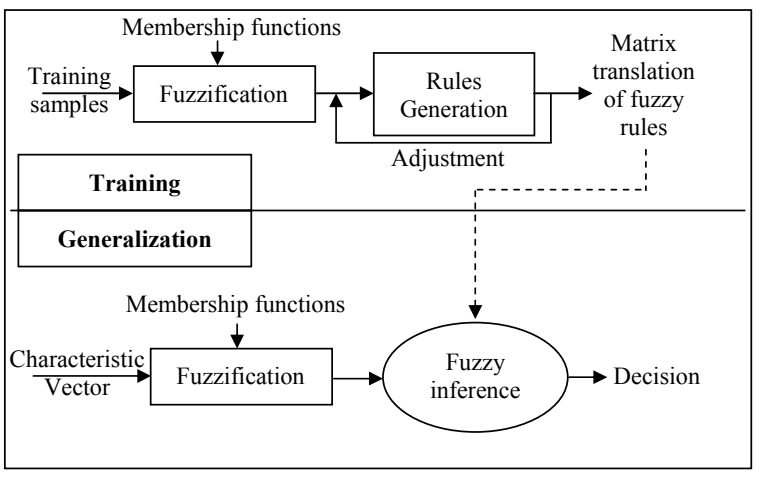

Fig. 9. Color recognition module

\subsection{Expert's knowledge integration}

Previously, we have presented two knowledge models, in the one hand a model for the wood field and the other hand for the vision field. To integrate the expert's knowledge into the numeric model, we must put in relation these two models. This part is in center of our fuzzy sensor notion (Fig. 6).

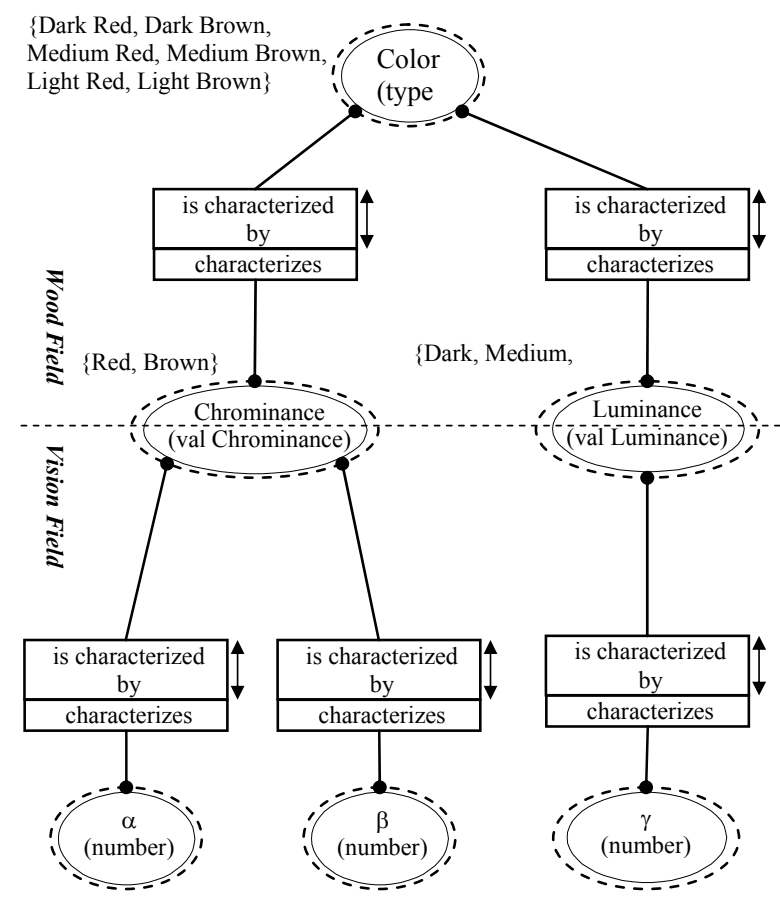

Fig. 10. Symbolic model for the color obtained from the two expertise fields
To realize this connection between the two fields, we put together the obtained symbolic models of wood expert knowledge and vision expert knowledge. Then, we represent the result following a new symbolic model (Fig. 10).

According to this symbolic model, we have a good knowledge of the useful parameters to create the characteristic vector used in our vision system. In the case of the color, we obtain the following vector $\mathrm{V}$.

$$
V=\left(\begin{array}{l}
m_{\alpha} \\
m_{\beta} \\
m_{\gamma}
\end{array}\right)
$$

where $\mathrm{m}_{\alpha}, \mathrm{m}_{\beta}$ and $\mathrm{m}_{\gamma}$ are the mean components of a colorimetric space.

Generally, the used colorimetric reference space is the one, which puts together the components Red, Green and Blue (RGB). But, the Commission Internationale de l'Eclairage (CIE) doesn't recommend the use of this space. In fact, these components are not independent in their representation space. This commission recommend spaces, which separate the chrominance and the luminance, as HIS (Hue-Intensity-Saturation), Yuv, Luv or Lab. Lots of studies have been made on these colorimetric spaces (Kline, et al., 1997). We have realized lots of tests to select the space, which gives the better color distribution. For confidential reasons, we don't precise the used colorimetric space.

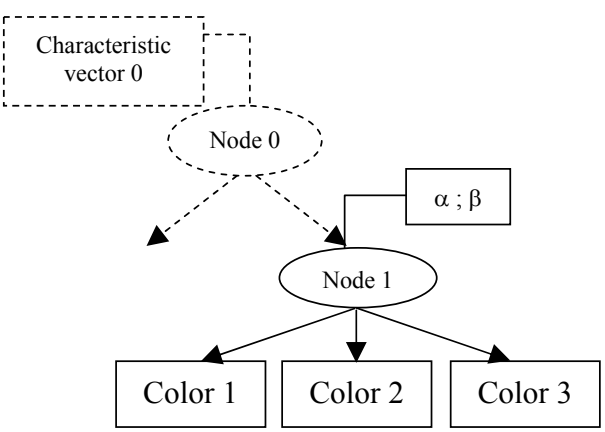

Fig. 11. Tree structure created thanks to knowledge model

Notice here that we can insert a notion of hierarchy in the attributes for our example. In fact, a color can be firstly characterized by the intensity level. Then, we can separate the different hues. Like this, we introduce the notion of tree structure in our numeric model (Fig. 11). The Fuzzy Inferences System, which we have realized, allows to interpret linguistically all inference engines of our model.

\section{COMPARATIVE RESULTS}

The set of realized tests use a batch composed of 1050 wooden samples for the generalization step of our methodology. The numeric model has been 
generated with 350 other samples. The results presented in the Table 1 concern the recognition rates of our method and the two following classifiers:

- a bayesian classifier;

- a kNN classifier (based on the " $\mathrm{k}$ Nearest Neighbour" theory) .

$\underline{\text { Table } 1 \text { Recognition rates of different methods and }}$ associated processing time

\begin{tabular}{cccc} 
& $\begin{array}{c}\text { Bayesian } \\
\text { Classifier }\end{array}$ & $\begin{array}{c}\mathrm{kNN} \\
\text { Classifier }\end{array}$ & $\begin{array}{c}\text { Fuzzy } \\
\text { Method }\end{array}$ \\
\hline Training & $82.67 \%$ & $86.13 \%$ & $89.86 \%$ \\
$\begin{array}{c}\text { Generalization } \\
\text { Time } \\
73.22 \%\end{array}$ & $79.01 \%$ & $83.12 \%$ \\
Processing & $120 \mu \mathrm{s}$ & $90 \mu \mathrm{s}$ & $50 \mu \mathrm{s}$ \\
\hline
\end{tabular}

The assets of our method are numerous for the color recognition on wooden boards. Firstly, by comparing our system versus more standard methods, we remark an improvement of the recognition rates for the training step and the generalization step. The second conclusion concerns the processing time. It is lower with our method. This is an important advantage for our system, because we have real-time constraints concerning the data processing.

As we have said before, our system is used on production lines, which may reach speeds of 180 meters of board length per minute. For a 8-hour day, 28000 meters of wood are scanned on average. That represents 28000 wooden pieces of 1 meter sorted in 8 hours, or 75 pieces per minute. A human operator can sort around 30 boards per minute, i.e. 14400 boards per production day. Thus, the use of our system allows to double the production with a certain stability in the classification.

\section{CONCLUSION AND PERSPECTIVES}

The color recognition on wooden boards is not universal; it is strongly linked to the samples used to generate the color model. Our numeric model is made up of a base of fuzzy conjunctive rules activated in parallel and merged disjunctively. It is created thanks to an automatic rule generation algorithm which replies to the constraints imposed by our system. This article describes the advantage of the fuzzy logic in the problem of color classification. It allows to take into account the color graduality. We must really take care of the acquisition conditions of the system. In fact, without a stable system in time, the models would be derived all days and the classification module would not recognize anything.

The second aspect of this study concerns the integration of expert's knowledge in our system. Then, we obtain a better coherence between the numeric model and the expert's knowledge model. The generated symbolic model allows to choose better the characteristic vector useful for each node of our fuzzy inferences system. The obtained recognition rates are encouraging for the following research. The last notion tackled in this paper is the development of our sensor following the fuzzy sensor concept (Maurice, et al., 1997). The data provided by this sensor type are symbolic information. These data are easier to interpret for the calibration of our production system.

We can use the fuzzy logic for other notions than the color. In fact, we want to develop an appearance fuzzy sensor putting together several attributes: the color and the texture. We have already created a knowledge model of the wood field concerning the texture. We must still determine the vision parameters to define the symbolic attributes explaining the texture characteristics.

\section{REFERENCES}

Benoit, E., Foulloy, L. (2000). High functionalities for intelligent sensors, application to fuzzy colour sensor. In: Measurement, Vol. 30, pp. 161-170.

Cordon, O., Del Jesus, M.J., Herrera, F. (1999). A proposal on reasoning methods in fuzzy rulebased classification systems. In: Int. Jour. Of Approximate Reasoning, Vol. 20, pp. 21-45.

Dubois, D., Prade, H. (1992). Fuzzy rules in knowledge-based systems - Modelling gradedness, uncertainty and preference. In: $A n$ introduction to fuzzy logic application in intelligent system. Kluwer, Dordrecht, pp. 4568.

Dubois, D., Prade, H. (1996). What are Fuzzy rules and how to use them. In: Fuzzy Sets and Systems, Vol. 84, pp. 169-185.

Galichet, S., Foulloy, L. (2003). Integrating expert knowledge into industrial control structures. Computer in Industry. Vol. 52, pp. 235-251.

Halpin, T.A. (1998). Object Role Modeling (ORM/NIAM). In: Handbook on Architectures of Information Systems.

Ishibuchi, H., Nozaki, K., Tanaka, H. (1992). Distributed representation of fuzzy rules and its application to pattern classification. In: Fuzzy Sets and Systems, Vol. 52, pp. 21-32.

Ishibuchi, H., Nozaki, K., Tanaka, H. (1997). A Simple but powerful heuristic method for generating fuzzy rules from numerical data. In: Fuzzy Sets and Systems, Vol. 86, pp. 251-270.

Kline, D.E., Conners, R.W., Lu, Q., Araman, P.A. (1997). Automatic color sorting of hardwood edge-glued panel parts. In: Hardwood Symposium Proceedings.

Klir, G., Yuan, B. (1995). Fuzzy Sets and Fuzzy Logic, Theory and Applications. Prentice Hall.

Maurice, G., Benoit, E., Foulloy, L. (1997). Fuzzy sensors: another view. In: Information Engineering.

Zadeh, L.A. (1973). Outline of a new approach to analysis of complex systems and decision processes. In: IEEE Trans. On Systems, Man and Cybernetics, Vol. 3, pp. 28-44.

Zimmermann, H.J. (1985). Fuzzy set theory and its applications. Dordrecht, Kluwer. 